\section{BMJ Open} Ophthalmology

\title{
Survey of the use of laser protective eyewear among international retina specialists: a European vitreoretinal society study
}

Mohammad Z Siddiqui, ${ }^{1}$ Abdallah A Ellabban (D) , ${ }^{2,3}$ Mohamed K Soliman (D) , ,,5 Giampaolo Gini, ${ }^{6}$ Shree Kurup, ${ }^{7}$ Sami H Uwaydat, ${ }^{1}$ Ahmed B Sallam (D) ${ }^{1}$

\section{ABSTRACT}

Objective To report the trends for the use of eye protection methods during retinal laser in clinic and operating room.

Methods and analysis Retrospective analysis of a 14-item survey questionnaire submitted to the European Vitreoretinal Society members.

Results Responses from 630 members were analysed. Most of the respondents practised in Europe (52.7\%), followed by North America (21.0\%). The majority of respondents had laser filters in the microscope for the operating surgeon $(92.1 \%)$, or used protective goggles (6.8\%). Only $38.9 \%$ of respondents indicated that auxiliary staff in the operative room used protective goggles during laser treatment. Three-dimensional retina viewing system was used by only $22.5 \%$ of respondents, of those, $34.5 \%$ reported use of laser protection goggles by the operating surgeon. Rates of laser protection by auxiliary staff were $62.9 \%$ for indirect laser and $60.8 \%$ for slit lamp laser. We found a higher rate for use of laser protection by auxiliary staff in North America-based practices for endolaser $(p<0.00001)$, laser indirect ophthalmoscope $(p<0.00001)$ and slit lamp laser $(p=0.00033)$ compared with the rest of the world.

Conclusion The use of laser protection methods is routinely adopted by the physicians in the operating room and clinic, but less so by their assisting or auxiliary staff.

${ }^{2}$ Department of Ophthalmology, Suez Canal University, Ismailia, Egypt

${ }^{3}$ Department of Ophthalmology, Hull University Teaching Hospitals NHS Trust, Hull, UK ${ }^{4}$ Ophthalmology, University of Ottawa Eye Institute, Ottawa, Ontario, Canada

${ }^{5}$ Ophthalmology, Assiut

University Faculty of Medicine, Assiut, Egypt

${ }^{6}$ Ophthalmology, Western Sussex NHS University Trust, Chichester, UK

${ }^{7}$ Department of Ophthalmology, Cleveland Clinic, Cleveland, Ohio, USA

Correspondence to Professor Ahmed B Sallam; ahmedsallam11@yahoo.com

\section{INTRODUCTION}

Laser for retinal photocoagulation or retinopexy is mainly performed using lasers within the blue-green light spectrum (488$532 \mathrm{~nm})$, as well as the red $(625-740 \mathrm{~nm})$ and yellow spectrum $(565-590 \mathrm{~nm})$. Delivery methods for retinal laser treatment include slit lamp, indirect ophthalmoscope and endolaser during the vitreoretinal surgery. The laser used for retinal treatment has a high output power range of up to $2 \mathrm{~W}$. By definition, this a class 4 laser that can cause retinal damage as a result of direct, diffuse or indirect beam viewing. These hazards may also apply to indirect or non-specular reflections of the laser beam. ${ }^{1-3}$

\section{Key messages}

What is already known about this subject?

While guidance on laser safety and use of protective eye wear exists, there is no literature on how retina specialists and their teams adhere to this guidance in clinic and theatre.

\section{What are the new findings?}

Use of laser protection methods is routinely adopted by the physicians in the operating room for endolaser use. However, only about $50 \%$ of their assisting staff in operating room or clinic adhere to laser guidance.

\section{How might these results change the focus of research or clinical practice?}

- The study is an eye-opener on how safety guidance for retinal laser use is practised in the real-world clinical setting. More research is needed to assess the reasons behind the lack of adherence to the laser guidance observed.

To control the risk of injury, various regulations have been put in place such as the 21 Code of Federal Regulations Part 1040 in the USA, British Standards Institution's laser equipment guidelines and the International Electrotechnical Commission 60825 in the rest of the world. ${ }^{45}$ These regulations impose required safety measures on manufacturers, such as labelling lasers with specific warnings, and enforce the use of the safety eye wear within the nominal ocular hazard distance (NOHD) when the laser is in operation. The NOHD is defined as the distance from the laser source up to where the level of radiant exposure is relatively safe (equal to the maximum permissible exposure), and varies according to the laser wavelength, beam divergence, laser energy output and mode of delivery. $^{23}$

While guidance on laser safety and use of protective eye wear exists, there is no literature 
on how retina specialists and their teams adhere to this guidance in real-word clinical practice. With this in mind, we sought to survey retina specialists through the European Vitreoretinal Society on their practices of eye protection during laser procedures using endolaser, slit lamp laser and laser indirect ophthalmoscope.

\section{METHODS}

After consultation with the European Vitreoretinal Society, a 14-item voluntary questionnaire was distributed electronically to all members in the database and three reminders were sent out. The questionnaire surveyed vitreoretinal specialists about the use of laser protection by in their practice including operation room and outpatient clinic. Questions were formulated mostly in a polar style (table 1). Patients or the public were not involved in the design, conduct, reporting or dissemination plans of this study.

We used multiple regression analysis to compare the use of laser protection between academic and private centres, and between regions of the world. We considered a $\mathrm{p}<0.05$ to be statistically significant. We performed statistical analyses using the SPSS-PC V.24 statistical package (SPSS).

\section{RESULTS}

Of the 1911 members contacted, 688 submitted responses to the questionnaire. Fifty-eight participants did not complete the responses for all the questions and were excluded. Left for analysis were a total of 630 respondents completed responses to all the questions $(33.0 \%$ response rate). The majority of respondents practised in Europe $(52.7 \%)$, followed by North America $(21 \%)$, Asia/Pacific (10.6\%), Africa/Middle East (10.6\%) and Latin America (5.1\%). Other subdividing factors considered were practice setting and years in practice. There was a split between private practice $(41.7 \%)$ and University/Government hospital (42.2\%). The remainder of the participants have mixed practice $(16.1 \%)$. The majority of the respondents $(93.5 \%)$ had been practising as retina specialists for greater than or equal to 5 years.

Table 1 summarises the results of the survey questions. Regarding endolaser protection during retinal surgery, $92.1 \%$ stated that their operating microscope has a protective laser filter for the operating surgeon; and $6.8 \%$ stated that they have no laser filter in the microscope, but the surgeon uses protective goggles. However, 7 of $630(1.1 \%)$ stated that their operating microscope did not have a laser filter, and the operating surgeon does not wear protective goggles. The majority of respondents $(64.3 \%)$, mentioned that the operating microscope has a protective filter for the surgical assistant. A smaller number of respondents $(28.7 \%)$ reported that there is no protective filter for the surgical assistant, but the assistant uses protective goggles; and only $7 \%$ stated that there is no protective filter for the surgical assistant, and the assistant does not use protective goggles. When surveyed about use of laser protection for auxiliary staff
Table 1 Responses of the 14-item questionnaire disturbed to the European vitreoretinal Society) members $(n=630)$ in relation to the trends for the use of eye protection methods during retinal laser

\begin{tabular}{|c|c|}
\hline Question & $\mathbf{N}(\%)$ \\
\hline \multicolumn{2}{|l|}{ Practice setting } \\
\hline \multicolumn{2}{|c|}{ 1. Which region of the world do you mainly practice? } \\
\hline Asia/Pacific & $67(10.6)$ \\
\hline Latin America & $32(5.1)$ \\
\hline North America & $132(21.0)$ \\
\hline Europe & $332(52.7)$ \\
\hline Africa/Middle East & $67(10.6)$ \\
\hline \multicolumn{2}{|c|}{ 2. Please list your main country of practice (free text) } \\
\hline \multicolumn{2}{|c|}{ 3. Which of the following best describes your main practice? } \\
\hline Private practice & $263(41.7)$ \\
\hline University or government hospital & $266(42.2)$ \\
\hline Mixed practice & $101(16.1)$ \\
\hline \multicolumn{2}{|c|}{ 4. How many years have you been in practice as a specialist? } \\
\hline $1-4$ years & $41(6.5)$ \\
\hline$>5$ years & $589(93.5)$ \\
\hline
\end{tabular}

\section{Endolaser}

5. Does your operating microscope have a protective laser filter for the operating surgeon?

Yes $580(92.1)$

No, but the surgeon uses protective laser $\quad 43(6.8)$ goggles

No laser filter, and the surgeon does not $\quad 7$ (1.1) use protective laser goggles

6. Does your operating microscope have a protective laser filter for the surgical assistant?

Yes 405 (64.3)

No, but the assistant uses protective laser 181 (28.7) goggles

No laser filter, and the assistant does not $\quad 44$ (7.0) use protective laser goggles

7. When performing endolaser during retinal surgery, do you use laser protective goggles for auxiliary staff in the operating room?

$\begin{array}{ll}\text { Yes } & 245(38.9) \\ \text { No } & 385(61.1)\end{array}$

\section{D vitreoretinal surgery}

8. When performing endolaser using the 3D surgery platform, do you use protective laser goggles for the operating surgeon?*

Yes $49(7.8)$

$\begin{array}{lr}\text { No } & 93(14.8) \\ \text { We do not perform 3D surgery } & 488(77.4)\end{array}$

9. When performing endolaser using the 3D surgery platform, do you use protective laser goggles for the surgical assistant and auxiliary staff?

$\begin{array}{lr}\text { Yes } & 60(9.5) \\ \text { No } & 82(13.1) \\ \text { We do not perform 3D surgery } & 488(77.4)\end{array}$

\section{Indirect laser retinopexy or PRP}

10. When performing indirect laser retinopexy or PRP, do you use protective goggles for auxiliary staff if they attend in the room?

$$
\text { Yes } 396 \text { (62.9) }
$$




\begin{tabular}{|c|c|}
\hline Question & $\mathrm{N}(\%)$ \\
\hline No & $234(37.1)$ \\
\hline \multicolumn{2}{|c|}{$\begin{array}{l}\text { 11. When performing indirect laser retinopexy or PRP, do you patch or } \\
\text { close the patient's other eye? }\end{array}$} \\
\hline Yes & $227(36.0)$ \\
\hline No & $403(64.0)$ \\
\hline \multicolumn{2}{|c|}{ Slitlamp laser retinopexy of PRP } \\
\hline \multicolumn{2}{|c|}{$\begin{array}{l}\text { 12. When performing slit lamp laser, do you use protective goggles for } \\
\text { auxiliary staff if they attend in the laser room? }\end{array}$} \\
\hline Yes & $383(60.8)$ \\
\hline No & 247 (39.2) \\
\hline \multicolumn{2}{|l|}{ General opinion } \\
\hline \multicolumn{2}{|c|}{$\begin{array}{l}\text { 13. What do you feel regarding the use of protective goggles in the } \\
\text { operating room? (with additional comments) }\end{array}$} \\
\hline Mandatory & $230(36.5)$ \\
\hline Unnecessary & $179(28.4)$ \\
\hline Equivocal/not sure & $221(35.1)$ \\
\hline \multicolumn{2}{|c|}{$\begin{array}{l}\text { 14. Have you ever witnessed or been made aware of any iatrogenic } \\
\text { laser injury to physicians or auxiliary staff while not wearing protective } \\
\text { goggles in your institution? If yes, please describe. }\end{array}$} \\
\hline Yes & $600(95.2)$ \\
\hline No & $30(4.8)$ \\
\hline
\end{tabular}

*Only 142 from 630 reported they use 3D vitreoretinal surgery.

3D, three dimensional; PRP, panretinal photocoagulation.

during endolaser in retinal surgery, $38.9 \%$ stated that their auxiliary staff routinely use protective goggles. Only 142 of 630 respondents $(22.5 \%)$ operated with 3 dimensional (3D) viewing system. Of those, 49 of the 142 $(34.5 \%)$ indicated the operating surgeon uses protective laser goggles during surgery; and only 60 of 142 (42.3\%) of the operating surgeons required their surgical assistants and auxiliary staff to wear laser protection goggles.

For laser protection during treatment with the laser indirect ophthalmoscope, our survey showed that $62.9 \%$ of the surveyed specialists required their auxiliary staff to wear protective goggles during the use of indirect laser retinopexy. A small number of the respondents, 36.0\% mentioned that the patient's fellow eye is routinely patched during the procedure for protection from laser. During the slit lamp laser, $60.8 \%$ of the retinal specialists stated that their auxiliary staff routinely wear protective goggles.

When asked about their beliefs of the necessity of routine use of laser protection measures, $28.4 \%$ stated they believed it is unnecessary; $36.5 \%$ thought laser protective goggles should be mandatory; and $35.1 \%$ were unsure or equivocal about the need for laser protection. The majority of the respondents, $95.2 \%$, have neither witnessed nor were made aware of any iatrogenic laser injury to physicians or auxiliary staff while not wearing protective goggles at their institution; but, $4.8 \%$ responded that they either witnessed or were made aware of a laser safety incident at their institution. Open responses to this question were also sought. Comments ranged from individuals stating that laser protective goggles are absolutely needed versus others stating it is not necessary but implemented for liability and regulatory reasons. Furthermore, one respondent described an iatrogenic laser injury due to a faulty laser filter during a slit lamp laser procedure.

We compared the use of laser protection between academic and private centres, and between regions of the world. We observed no significant difference between academic vs private practices for adoption of laser protection during slit lamp laser or laser indirect ophthalmoscopy $(\mathrm{p}=0.1564,0.2085$, respectively). However, with endolaser, University/Government hospitals reported a higher use of laser protection for auxiliary staff compared with private practice and mixed practice settings $(\mathrm{p}=0.00186$ and 0.0057 , respectively). Regarding the adherence to laser guidance by auxiliary staff, we found the use of safety goggles to be higher in North American centres as compared with Europe during the endolaser $(76 / 132,57.6 \%, \mathrm{p}<0.0001$ vs $110 / 332,33.1 \%$, $\mathrm{p}=0.0009$, respectively) and for laser indirect ophthalmoscope use $(110 / 132,83.3 \%$, p $<0.0001$ vs $189 / 332,56.9 \%$, $\mathrm{p}=0.0004$, respectively, figure 1$)$. There was also a higher rate of laser protection use by auxiliary staff during slit lamp laser procedures in North America (244/332), $73.5 \%, \mathrm{p}=0.0001$ ).

\section{DISCUSSION}

This survey found that in the clinical setting, almost all retina physicians adopted the recommended safety guidelines when performing endolaser. However, laser protection practices were not uniformly followed by the surgical assistant or auxiliary staff in the clinic and the operating room, and the results varied between the different regions of the world.

Our survey showed that when operating with a microscope, nearly all surgeons were compliant with laser protection guidelines when endolaser was in use. For 3D surgery, we found that only half of the surgeons use laser protection goggles. This may stem from that there are currently no clear recommendations on laser protection for the surgeons when operating with 3D surgery and not directly looking through the microscope. It is plausible to presume that compared with the microscope oculars, the screen projection of the microscope image in 3D surgery does not emit any significant laser radiation to the surgeon's eyes, and has a negligible risk of laser scatter. Another possible reason for not using laser protection during 3D surgery is that using the laser eyewear on top of the 3D glasses may disrupt 3D viewing; however, this could be mitigated by reversing the arrangement of the laser eyewear in relation 3D glasses. ${ }^{6}$ It is of note that the number of respondents who use 3D surgery was relatively small, which is not surprising given that the technology is relatively new.

We observed that a substantial proportion of the auxiliary staff in the operating room, and during indirect and slit lamp retinal laser procedures do not adhere to the 


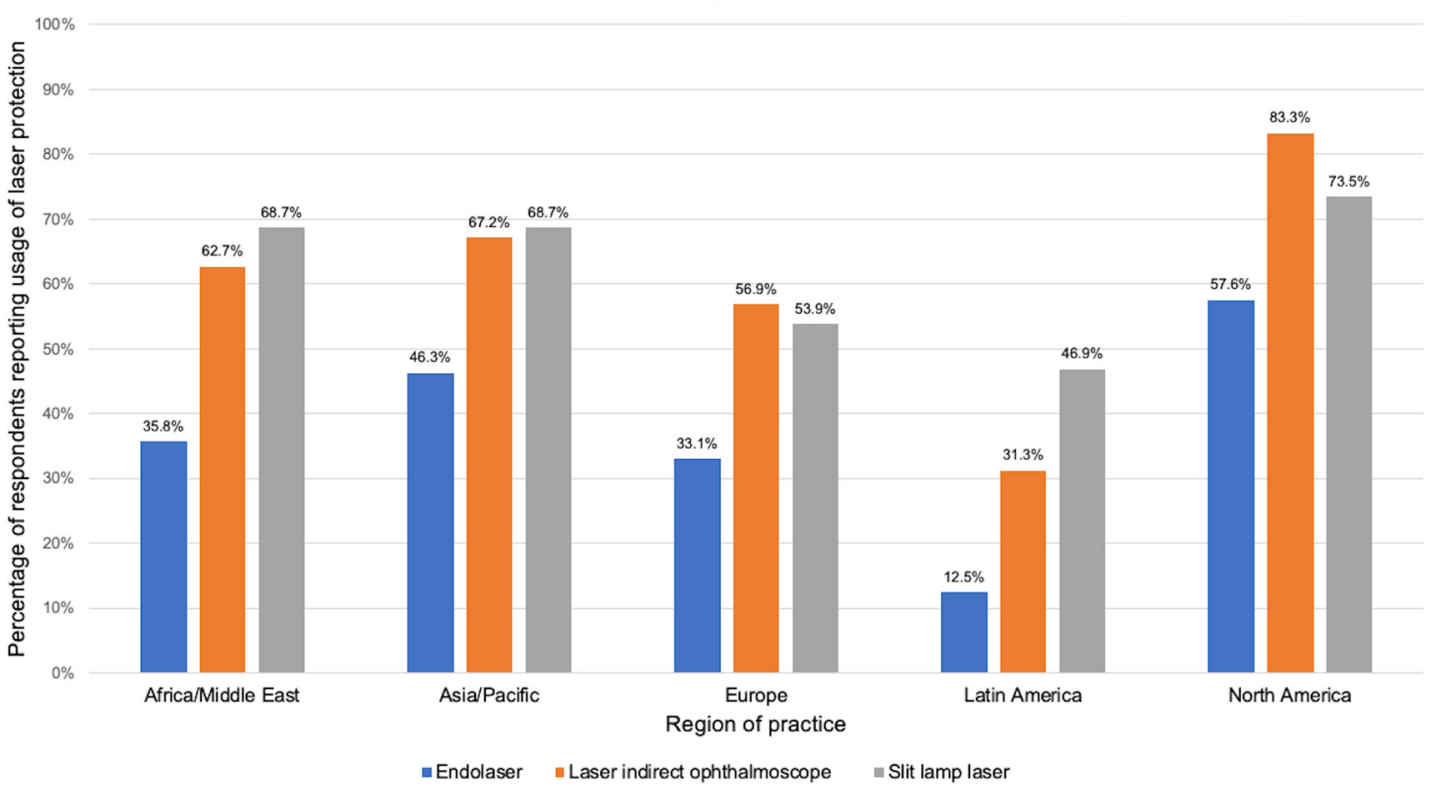

Figure 1 Laser protection use by auxiliary staff for different types of retinal laser divided by region of practice.

recommended laser protection guidance. Only about $40 \%-60 \%$ of the respondents reported that their auxiliary staff routinely use laser protection goggles in these settings, with the uptake being higher in North American practices compared with the other regions of the world (figure 1). While laser injury is less likely to occur if a person is further away from the laser source or if low laser energy is used, the NOHD has been estimated to be in the range of approximately $20 \mathrm{~m}$ for the laser indirect ophthalmoscope and $3 \mathrm{~m}$ for retinal endolaser within the blue-green light spectrum. ${ }^{23}$

Although rare, ocular injury from retinal laser in the absence of laser protection eyewear could occur in few scenarios. First, most laser-induced retinal injuries are caused by accidental laser discharge during the preparation of laser devices with the medical personnel directly looking at the source of laser without protection. ${ }^{78}$ Second, damage to the laser equipment might result in unintentional emergence of radiation, for example from a damaged endolaser probe or faulty laser filters as reported in this survey. Finally, psychosocial issues of the surgeons may be a potential cause for laser injury. Studies have shown that a small number of surgeons may exhibit a disruptive behaviour towards colleagues which may include verbal and physical violence. ${ }^{9}{ }^{10}$ In a qualitative study that analysed surgeon behaviour in the perioperative environment by, Cochran and Elder, reported surgeons directly throwing objects at colleagues among other patterns of abusive behaviour in the in the operating room. ${ }^{9}$ Although a deliberate error involving the use of laser towards coworkers has not been reported, it could still occur.

Safety concepts and measures in the healthcare system have largely been adopted from the aviation industry. ${ }^{11}$ To safeguard safety standards in aviation, there is strong emphasis on root cause analyses and constructive learning from adverse events, including those that are rarely encountered. Along the same line of reasoning, the fact that accidental injury from retinal laser is rare, should not make the medical personnel reluctant to adhere to the recommended laser protection policies, as the consequences of sustaining a retinal injury are dire to the person and their organisation. Therefore, it is important to take all required safety measures during laser treatment, in addition to eye protection, such as putting the laser in standby mode when not in use, turning the laser hazard light and closing the operating room door while laser is in use.

The results of this survey need to be interpreted with caution. Similar to other survey studies, there may be bias due to the voluntary nature of this survey and bias due to the sample size. Another limitation is that our results are more reflective of the world region of practice rather than specific countries, given the small number of responses from individual countries. Also, because this questionnaire is the first of its kind, there is limited literature to compare to in ophthalmology. Despite these limitations, our study is a useful examination of how retina specialists and their teams adhere to existing guidance on laser safety in the current real-word clinical setting.

We conclude that the use of laser protection by the operating retinal physicians during endolaser is reassuring. However, laser protection is not uniformly adopted by the auxiliary staff in the clinic or in the operating room. More research is needed to assess the reasons behind this trend.

Contributors AAE (conception, design, critical appraisal, writing the article, final approval of the article), MZS (writing the article, statistical analysis, final approval of the article), MKS (critical appraisal, writing the article), GG (conception, critical appraisal, final approval of the article), SHU (critical approval, final approval), AS (conception, design, critical appraisal, writing the article, final approval of the article). 
Funding The authors have not declared a specific grant for this research from any funding agency in the public, commercial or not-for-profit sectors.

Competing interests None declared.

Patient and public involvement Patients and/or the public were not involved in the design, or conduct, or reporting, or dissemination plans of this research.

Patient consent for publication Not required as no patient involvement.

Ethics approval The study adhered to the Declaration of Helsinki; because the study did not involve any patients' data, an ethical committee approval or exemption was not required.

Provenance and peer review Not commissioned; externally peer reviewed.

Data availability statement All data relevant to the study are included in the article.

Open access This is an open access article distributed in accordance with the Creative Commons Attribution Non Commercial (CC BY-NC 4.0) license, which permits others to distribute, remix, adapt, build upon this work non-commercially, and license their derivative works on different terms, provided the original work is properly cited, appropriate credit is given, any changes made indicated, and the use is non-commercial. See: http://creativecommons.org/licenses/by-nc/4.0/.

ORCID iDs

Abdallah A Ellabban http://orcid.org/0000-0002-6033-2969

Mohamed K Soliman http://orcid.org/0000-0003-1671-8925

Ahmed B Sallam http://orcid.org/0000-0001-7207-6782

\section{REFERENCES}

1 ANSI. American national standard for safe use of lasers in health care, 2018: 28-30.

2 Delori FC, Webb RH, Sliney DH, et al. Maximum permissible exposures for ocular safety (ANSI 2000), with emphasis on ophthalmic devices. J Opt Soc Am A Opt Image Sci Vis 2007;24:1250-65.

3 Smalley PJ. Laser safety: risks, hazards, and control measures. Laser Ther 2011;20:95-106.

4 British standards Institution. Safety of laser products. equipment classification and requirements (IEC 60825-1), 2017.

5 International Electrotechnical Commission (IEC). "Safety of laser products," IEC 60825, 2001.

6 Duddleston J, Chancellor JR, Ellabban AA, et al. Optical principles in three-dimensional vitreoretinal surgery and laser eyewear. Ophthalmol Retina 2020;4:224-5.

7 Lee YH, Kim YC. Foveal choroidal neovascularization secondary to accidental laser exposure in a dermatologist: a case report. Medicine 2019;98:e15429.

8 Barkana Y, Belkin M. Laser eye injuries. Surv Ophthalmol 2000;44:459-78.

9 Cochran A, Elder WB. A model of disruptive surgeon behavior in the perioperative environment. J Am Coll Surg 2014;219:390-8.

10 Halverson AL, Neumayer L, Dagi TF. Leadership skills in the or: Part II: recognizing disruptive behavior. Bull Am Coll Surg 2012;97:17-23.

11 Hudson P. Applying the lessons of high risk industries to health care. Qual Saf Health Care 2003;12:7i-12. 